\title{
Hydrophobic Detection Study of Composite Insulator Based on BP Neural Network Grading
}

\author{
Bo Wang ${ }^{1,2, *}$, He Ninghui ${ }^{2}$, Shi Tao Liu ${ }^{2}$ and Wanghai Long ${ }^{2}$ \\ ${ }^{I}$ School of Electrical Engineering Wuhan University, Hubei, Wuhan, 430072, China \\ ${ }^{2}$ Ningxia Electric Power Research Institute, Ningxia, Yinchuan, 750002, China
}

\begin{abstract}
With the rapid development of industry and agriculture in our country, the atmospheric contamination degree is intensifying, which has become the main factors of influencing the electrical equipment insulation level. In order to ensure the security of system operation, the periodic hydrophobic detection of the composite insulator in the operation is required to determine its safety. According to the existing problem of low detection accuracy of the present composite insulator hydrophobic detection, this paper proposes a BP neural network grading based composite insulator hydrophobic detection model. The model is optimized and improved on the basis of the traditional BP neural network algorithm and carries out positive and negative transmission after input samples, then compares tonsure weight and the size of the error function, calculates according to the comparison results, adjusts the weight of nodes and results in error reduction of the traditional BP neural network algorithm. Experimental simulation results show that BP neural network grading based composite insulator hydrophobic detection model is superior to transitional BP neural network algorithm on convergence and network error, which can be well applied into the composite insulator hydrophobic detection.
\end{abstract}

Keywords: Composite Insulator, Hydrophobic Detection, BP Neural Network, Grading Operation.

\section{INTRODUCTION}

With the rapid development of industry and agriculture in our country, the atmospheric contamination degree is intensifying, which has become the main factors of influencing the electrical equipment insulation level [1]. Electric power system has experienced from the porcelain insulator to the glass insulator and finally silicone rubber is utilized as composite insulator, which is chosen as the main insulator to improve the antifouling flash capacity of transmission lines. Hydrophobic nature is a kind of physical property of low surface energy solid material. Silicone rubber surface has good hydrophobic property, namely after the insulator surface is affected by damp, the adsorbed moisture exists in the form of discrete isolated small water droplets and does not form a continuous water film. It also limits the surface leakage current and increases the flashover voltage $[2,3]$. The operating experience also suggests that silicon rubber's external insulation and the hydrophobic mobility do not remain constant [4]. To test the hydrophobic properties of the composite insulator is the important means of judging composite insulator's pollution flashover resistance; which is also an important guarantee to ensure the safe operation of the composite insulator. Therefore, it is very important to carry out the study of composite insulator's hydrophobic detection technology.

*Address correspondence to these authors at the School of Electrical Engineering Wuhan University, Hubei Wuhan, 430072, China;

E-mail: 232464433@qq.com
Currently, the hydrophobic detection of composite insulator mainly includes static water contact angle method, blow grading method and hydrophobic indicator function method. The static water contact angle method and blow grading method are traditional detecting methods, which are suitable for measurements in the laboratory [5-7]. Hydrophobic indicator function utilizes digital camera technology and computer digital image processing technology to judge the insulator hydrophobic nature, its essence is using nonlinear mathematical method to calculate and evaluate insulator hydrophobic [8]. Hydrophobic indicating function method has been developed very rapidly in recent years. It can be subdivided into many methods, the most typical are entropy method and shape factor method [9]. Hydrophobic indicating function method overcomes the disadvantages of complex measuring process, strict conditions demanding, and has only confined to laboratory operations of traditional detection method, so it has attracted the attention of large groups of scientific and engineering professionals [10-12]. In addition to the above methods, we can also show directly by leakage current of wet environment to indicate the hydrophobic of composite insulator. Some researchers have tried to describe sizing of the composite insulator's hydrophobic nature by measuring the high polymer surface charge ability, such as COV method proposed by Gorur, but is rarely used in the site operations. Marcus has proposed the assumption of using helicopters to carry out line composite insulator's hydrophobic charged detection, but this technique is extremely expensive [13]. 


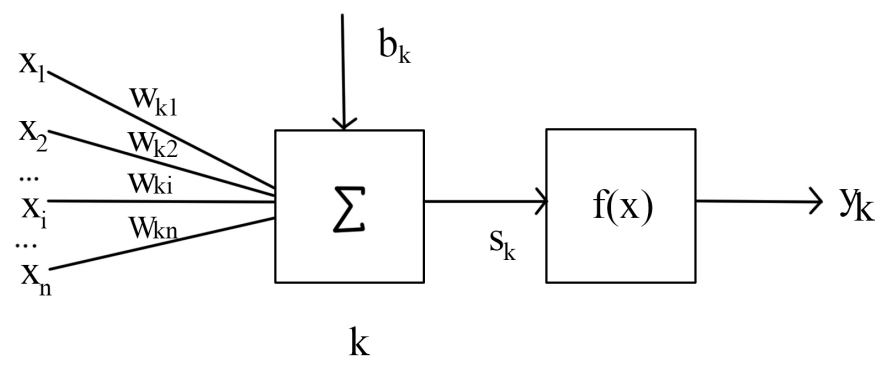

Fig. (1). BP Neuron model.

Based on the traditional hydrophobic detection method of composite insulator, this paper proposes an improved BP neural network algorithm based on composite insulator hydrophobic detection scheme, which greatly improves the accuracy of hydrophobic composite insulators detection.

\section{BP NEURAL NETWORK ALGORITHM}

\subsection{BP Neural Network Summary}

BP (Back Propagation) network is proposed by a group of scientists headed by Rumelhart and McCelland in 1986, which is a multilayer feedforward network trained by error Back Propagation algorithm. It is one of the most widely used neural network models. BP network can learn and store a lot of input - output model mapping relations, without prior to revealing mathematical equations that describe the mapping relations. Its learning rule is to use the steepest descent method, and constantly adjust the network weights and thresholds by back propagation and minimization of the error sum of squares of the network. Topological structures of BP neural network includes input layer, the hidden layer and output layer $[14,15]$.

BP neural network algorithm is proposed on the basis of existing algorithm of BP neural network, which means arbitrary selection of a set of weights, output the given target directly as algebraic sum of linear equation to establish the linear equations, solve the weight, thus there is no problem of local minimum and slow convergence speed of traditional methods, and is easier to understand.

\subsection{BP Neural Network Structure}

In biology, the neural is referred to the nerve cells, which is the structure and function unit of nervous system. The neurons have the function of feeling stimulus and conducting exiting. The neurons of neural networks imitate the three basic functions of biological neurons: weighting, summation and transfer, and BP neuron is similar to other neurons. The difference is that the BP neuron's transfer function is nonlinear function, more commonly used functions are logsig, tansig and purelin $[16,17]$.

Fig. (1) gives the normal model of BP neural network, in which $x_{1}, x_{2} \cdots x_{n}$. respectively represent the input of neurons $1,2 \cdots n ; W_{k 1}, W_{k 2} \cdots W_{k n}$ respectively indicate the neuron $1,2 \cdots n$ and the joint strength of neuron $\mathrm{k}$, namely the weight value; $b_{k}$ is for the threshold; $f_{(x)}$ is for the transfer function; $y_{k}$ is for the output of neuron $k$. The net input value $B_{k}$ of neuron $k$ is shown in formula (1):

$B_{k}=\sum_{i=1}^{n} w_{k 1} * x_{1}+b_{k}=W_{k} X+b_{k}$

Topology of neural network represents connection patterns of neurons, according to different connection mode, the network can be divided into feedback type of network (also called circular network) and the non-feedback network. The feedback type network refers to the feedback loop between neurons; while in the non-feedback network, if neurons are hierarchically arranged, and each layer of neurons is connected only with the previous layer of neurons, thus this kind of network is called a feed forward network. Feed forward networks can be divided into several layers, each layer only receives the information from the previous layer, input messages are delivered to only one layer in turn, which constitutes the multilayer feed forward neural network, which is the BP neural network. Fig. (2) is the BP neural network structure model, divided into input layer, hidden layer and output layer. The hidden layer can be a single layer or multilayer. Sample information $x_{1}, x_{2} \cdots x_{n}$ is delivered from the input layer through the hidden layer, until to the output layer. In the deliver process, each layer of neurons only affects its next layer of neurons.

The comparison of the actual output and desired output is carried out, if error is bigger than the neural network allowed error precision then it is entered into back operation process. The back operation reversely returns the error signal according to the original forward operation path simultaneously modifying each neuron's weight in i in hidden layer, making the expected error signal to a minimum and in the end, $y_{1}$, $y_{2} \cdots y_{n}$. as a output result of the neural network.

\subsection{BP Neural Network Learning}

After determining the structure of BP neural network, it needs to train the network through the input and output sample set, namely study and modify the threshold and weight of 


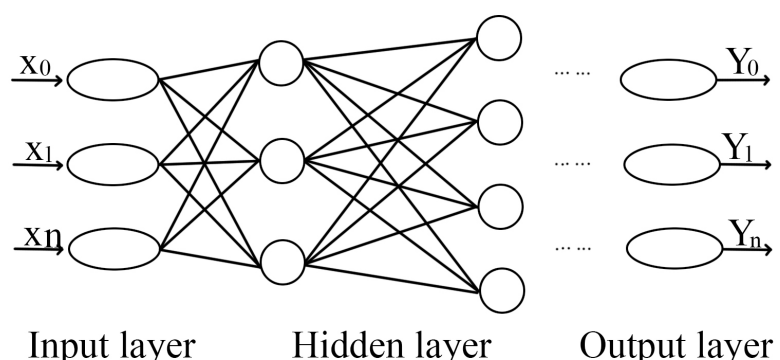

Fig. (2). BP neural network structure.

neural network, making the network achieve the given input/output mapping relationship, which is the learning of BP neural network. The standard BP algorithm is a kind of gradient descent learning algorithm; the weight correction is along the opposite direction of the error performance function gradient. Aiming at the shortcomings of the standard BP algorithm, some improved algorithms are given below:

1. Fastest descend BP algorithm

2. Assume $k$ is for the iteration time, then the modification of each layer's weight and threshold proceeds according to the following formula:

$x(k+1)=x(k)-\alpha g(k)$

In which: $x(k)$ is for the connected weight vector or threshold vector between each layer at $k$, s iteration:

$g(k)=\frac{\partial E(k)}{\partial x(k)}$ is for the gradient vector of neuron network output error on each weight or threshold at $k$,s iteration. ${ }^{a}$ is for the learning rate, which is a constant at training.

$E_{k}$ is for the performance function of network output error at $k$ 's iteration.

The advantage of this improved algorithm is that we can get relatively stable weight and threshold values. If the convergence speed is slow, the network is easy to fall into local extremum, thus oscillations occur in the learning process.

(2) Momentum BP algorithm

Momentum BP algorithm introduces momentum factor $\eta(0<\eta<1)$ on the basis of gradient descend algorithm:

$\Delta x(k+1)=\eta \Delta x(k)+\alpha(1-\eta) \frac{\partial \sum(k)}{\partial x(k)}$

$x(k+1)=x(k)+\Delta x(k+1)$

This algorithm determines the second correction effect based on the previous modification effect and thus making the correction amount increase in the same gradient direction. If the previous correction amount is too large, the second symbol of type (3) is in contrast to the previous correction symbols; If the previous correction amount is too small, the second symbol of type (3) is the same to the previous correction symbols. Compared with the steepest descent BP learning algorithm, momentum BP learning algorithm shows a better convergence effect, which has a shorter learning time.

\section{IMPROVED BP NEURAL NETWORK ALGO- RITHMS}

\subsection{Improvement Ideas of BP Neural Network Algo- rithm}

In the back propagation stage of BP neural network, the connection weights between different nodes use different learning rate to carry out optimization search. For example,

in reverse adjustment of the connection weight $w_{j p 1}, w_{j p 2}, \ldots, w_{j p n}$ between the output layer $P$ and hidden layer $J$, using learning rates $\eta_{1}, \eta_{2}, \ldots, \eta_{n}$ to adjust. The differences in back adjustment between different nodes can maximally mobilize the learning rate adaptability, maximally satisfies the changing requirements of weights in the study. After improvement, the back propagation figure of adaptive adjustment is shown in Fig. (3).

The random selection of small $\eta$ as the initial learning rate is carried in the improved learning adaptive algorithm. In searching for the maximum of error function $E(w)$ :

(1) When $E[w(n+1)]<E[w(n)]$, it will keep the original searching direction. If step is longer it will keep on learning. The new step $\eta^{*}$ can be obtained from the following formula.

$2 \times \eta \Rightarrow \eta^{*}$

(2) When $E[w(n+1)]>E[w(n)]$, at this time, it only needs to shorten the step, keep learning in the reverse direction of the original searching direction. The new step $\eta^{*}$ can be calculated from the following formula. 


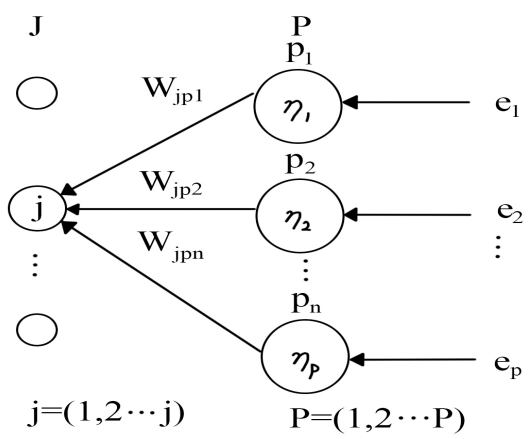

Fig. (3). Reverse the adjustment process to improve the learning rate adaptive algorithm.

$\eta \div 2 \Rightarrow \eta^{*}$

\subsection{Improved BP Neural Network Algorithm Process}

Applying new learning rate adaptive algorithm to carry out the learning of BP neural network, it needs to be implemented according to the derivation of the algorithm and through computer programming. The improved BP neural network can be learnt according to the following steps.

(1) BP neural network's initialization. Set the network structure, determine the expectations of sample input and sample output. Randomly select the smaller weight matrix $W$ and learning rate $W$, and set error precision of network learning $e$.

(2) Input samples, forward-propagating. Record the error $E^{(0)}$ of this forward propagation.

(3) Counter-propagation, adjust weights. For reversed order weight matrix $W^{*}=\left[w_{1}, w_{2}, \ldots, w_{n}, \ldots, w_{m}\right]$, select the vector $\Delta w_{i}(n), i=1,2, \ldots, m$ step by step to adjust, if the gradient of error function on weight is less than the preset minimum $\varepsilon$, namely $\frac{\partial E}{\partial w_{i}}<\varepsilon$, then turn to step (5). If the gradient is larger than the preset minimum, namely $\frac{\partial E}{\partial w_{i}}>\varepsilon$, then turn to step (4).

(4) Find the optimal weights in the gradient direction. First adjust the weights and forward propagation calculation error $E^{(1)}$. Compared $E^{(1)}$ with $E^{(0)}$, if network error decreases then increase learning rate, and readjust the weights, and forward propagation calculation $E^{(1)}$, until there is no longer reduction in error. If online learning rate increases, readjust the weights, and calculate forward propagation calculation $E^{(1)}$, until error no longer reduces.

(5) Using new weights for forward propagation, update the network error value $E^{(1)}$. If error is less than the preset accuracy $e$, the network jumps out to enter propagation, end the study, and enter the step (6). If the error is greater than the preset accuracy $e$, order $i=i+1$, when $i<m$, namely there are nodes' weight that have not been adjusted, then go to step (2) to add a weight adjustment. When $i=m$, that is, all the weights between nodes have been adjusted but the error at this time does not meet the precision requirement, then order $i=0$, carry out a new iterative learning since the first node.

(6) End the BP neural network learning and record the final weight matrix.

\section{ALGORITHM PERFORMANCE SIMULATIONS}

In order to explore the improved effect of BP neural network algorithm, we carried out the simulation experiments and compared with the traditional BP neural network algorithm.

\section{(1) Sample data processing}

Input sample data as $1.5 \pi \sim \pi$, input interval is $0.05 \pi$, the total number of samples is 50 , the size of the sample data is in the range of $(-4.3,7.2)$. In order to avoid the derivative flat change area of Sigmoid function, this article compresses the sample data into the range $(0.2,0.8)$. If set $X^{*}$ for the sample after data processing, $X_{\min }$ and $X_{\text {max }}$, is respectively the minimum and maximum values of the original sample data; the sample can be processed according to the following type.

$$
X^{*}=0.2+0.6 \times \frac{\left(X-X_{\min }\right)}{X_{\text {max }}-X_{\text {min }}}
$$

(2) Determine the BP neural network structure, set the initial parameters.

Under the provisioning error precision of 0.0001 , random selection of initial vector and the weights in the interval $[0,1]$, using Sigmoid function as the activation function. A number of studies have shown that a continuous function can be approximated by using a network in a hidden layer of any closed area. So this experiment selects a BP neural network 


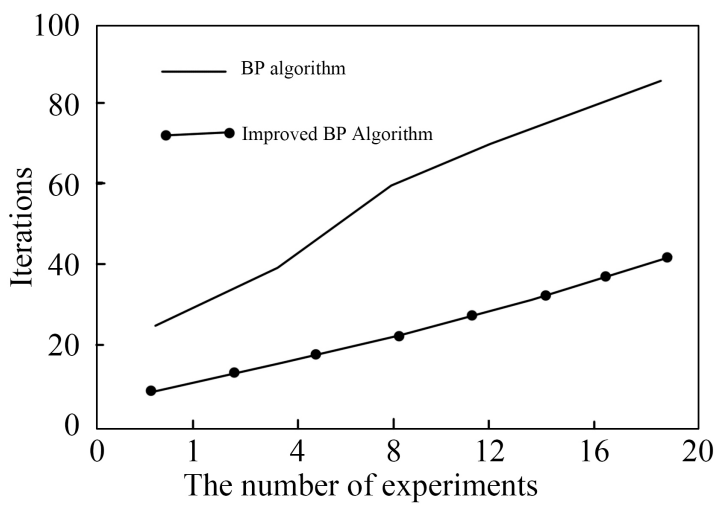

Fig. (4). Convergence rate comparison chart.

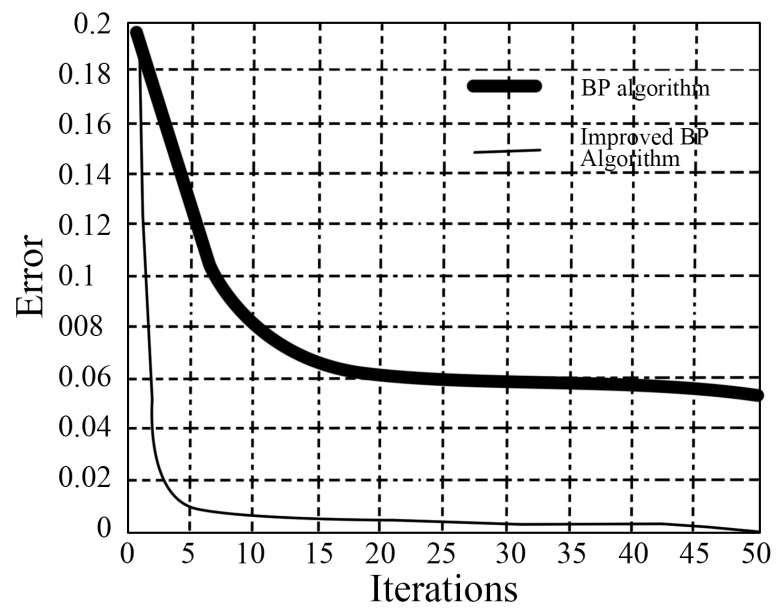

Fig. (5). Error contrast experimental results.

structure in a hidden layer to learn. The functions fitting problem requires only one unit in neural output layer, namely $O=1$. Considering that the test sample size is enough, we set the number of input layer nodes to 5, i.e. $I=5$. Take constant $a=6$, and calculate hidden layer nodes $H=9$. Then the network structure of this experiment is ultimately determined as: a hidden layer BP neural network of 5-9-1.

(3) Carry out computer programming calculation for the new algorithm of improved BP neural network.

(4) Record the experimental results of new algorithm and compare to analyze the learning performance of improved BP neural network.

This article takes the average value of 20 times computing as a test result. The compared figure of convergence speed of improved BP neural network algorithm and traditional BP neural network algorithm is shown in Fig. (4).

Then error statistic of improved BP neural network algorithm and the traditional BP neural network algorithm is carried out. The network error curve is shown in Fig. (5).

We can clearly found that after 5 times of learning, the improved BP neural network algorithm's error can be decreased to around 0.01 . Since then, after 40 times of iterative learning, the network can meet the requirements of 0.0001 error precision, the error convergence speed is fast. Simultaneously, the error curve is smooth, which does not appear obviously to beat phenomenon; the entire network learning process is fast and smooth. Its network error is lower than the traditional BP neural network algorithm. By using the proposed improved BP neural network algorithm to detect composite insulator hydrophobic can make a better convergence performance than the traditional BP neural network, and the network error is smaller.

\section{CONCLUSION}

Digital image processing techniques are often used in the preprocessing of composite insulator hydrophobic image, which contains digitization, filtering denoising, image segmentation, and target recognition process. The particularity and complexity of hydrophobic image as compared to traditional image processing algorithms cannot successfully complete image pretreatment process. This paper proposes an improved BP neural network algorithm based on composite insulator hydrophobic detection method. The experimental simulation results validate that the proposed method is effective and worthy of spreading-utilization. 


\section{CONFLICT OF INTEREST}

The authors confirm that this article content has no conflict of interest.

\section{ACKNOWLEDGEMENTS}

Declared none.

\section{REFERENCES}

[1] H. P. Chen, W. H. Sheng, N. Xi, and M. Song, "Automated robot trajectory planning for spray painting off free form surfaces in automotive manufacturing," IEEE International Conference on Robotics and Automation, USA: Washington DC, vol. 5, pp. 450-455, 2002.

[2] Y. H. Tang, and X, Y, Liu, "Reliability analysis of one unit repairable system with repairman vacation," Acta Automatica Sinica, vol. 3, pp. 466-470, 2004.

[3] T. Y. Hao, 1. W. Shu, and C. Zhen, "A phase matching method for the stepped chirp phased array radar," In: IET International Radar Conference, pp. 1-4, 2009.

[4] W. Liu, S. Weiss, and L. Hanzo, "A subband-selective broadband GSC with cosine-modulated blocking matrix," IEEE Trans, Antennas and Propagation, vol. 8, pp. 813-820, 2004.

[5] A. S. Stava, A. Kumar, and A. Singru, "Design and analysis of a video-on-demand server," Multimedia Systems, vol. 5, pp. 238-253, 1997.

[6] Y. L. Zhang, "Geometric process and analysis of a cold standby system," Journal of the Central University for Nationalities, vol. 1, pp. 15-22, 1995.
[7] Y. H. Tang, and X. Y. Liu, "The cold standby repairable system with not be good as new and repair man vacation," Journal of Mathematics in Practice and Theory, vol. 38, pp.47-52, 2008.

[8] H. Mir, and Z. Berkowitz, "Sub-band STAP for stretch processed systems," IEEE International Conference on Acoustics, Speech and Signal Processing, pp.2025-2028, 2009.

[9] H. T. Liu, X. Y. Meng, and W, J, Wu, "The cold standby system with repair of non-new and repairman vacation," Journal of Computational Information Systems, vol. 8, pp.1349-1357, 2012.

[10] Q.Y. Sun, L.Q. Ye, and C. X. Xu, "Global convergence of modified GLP gradient projection method," Journal of Engineering Mathematics, vol. 6, pp. 95-100, 2003.

[11] S. Babaie - Kafaki, "A modified BFGS algorithm based on a hybrid secant equation," Science China(Mathematics),vol. 9, pp.20192036, 2011.

[12] Q. Tang, and L, Chen, "Extended kci attack against two-party key establishment protocols," Information Processing Letters, vol. 111, pp.744-747, 2011.

[13] W. J. Cheng, H, Q, Li, X, Ruan, "Modified Quasi-Newton Algorithm for Training Large-scale Feed forward Neural Networks and Its Application," Journal of Computational Information Systems, vol. 9, pp.3047-3053, 2011.

[14] J. Yang, and T. Cao, "A verier-based password-authenticated key exchange protocol via elliptic curves," Journal of Computational Information Systems, vol. 7, pp.548-553, 2011.

[15] D. L. Jia, and S. Z. Jia, "Niche particle swarm optimization combined with chaotic mutation," Control and Design, vol. 22, pp.117120, 2007.

[16] C. T. Lawarence, and A. L. Tits, "A computationally efficient feasible sequential quadratic programming algorithm," SIAM Journal of Optimization, vol. 4, pp. 1092-1118, 2001,

[17] C. K. Wang, "Ocean energy knowledge lecture Characteristics of ocean energy," Solar Energy, vol. 10, pp. 32-35, 2008.

Received: May 26, 2015

(C) Wang et al.; Licensee Bentham Open.

This is an open access article licensed under the terms of the (https://creativecommons.org/licenses/by/4.0/legalcode), which permits unrestricted, non-commercial use, distribution and reproduction in any medium, provided the work is properly cited. 